\title{
Dead-beat functional observers for discrete-time LPV systems with unknown inputs
}

\author{
Mirko Fiacchini*, Gilles Millerioux ${ }^{\#}$
}

\begin{abstract}
This paper deals with functional observers for discrete-time Linear Parameter Varying (LPV) systems. More specifically, dead-beat observers for reconstructing linear combinations of the state are proposed. The system inputs are assumed to be unknown and the observers reduce to functions of the output over a finite number of time-steps. The existence of such observers is proven by resorting to the notion of inverse system together with the concepts of maximal robust invariant subspaces and nilpotent semigroups. A constructive approach to derive the explicit equations of the observer is provided. An example illustrates the efficiency and the computational aspects of the method.
\end{abstract}

\section{INTRODUCTION}

Reconstructing the state vector of a system from its accessible outputs is an important issue in automatic control. In various contexts, the inputs of the system may be unknown, either because the control is not accessible as in some decentralized control setups or because the inputs actually stand for disturbances and faults, unknown by definition. Functional observers aim at reconstructing a particular (often linear) function of the state (possibly of the input as well). Their use is motivated by several arguments. Determining a particular set of states can be especially interesting for diagnosis, fault detection and isolation problems or for supervision. Indeed, in this context, one can focus on a restricted set of state variables. Furthermore, partial state reconstruction can be well suited to face computational and real-time stringent constraints, as often required for embedded or large-scale systems. Finally, functional observers are useful to directly estimate a feedback control without estimating each component of the state.

Functional observers for linear systems have been widely addressed since the pioneering work [18]. A complete framework providing necessary and sufficient conditions for convergence and design procedure has been presented in [12]. The books [10] and more recently [30] present a state of the art of the research in this field. Functional observers have been investigated for some classes of nonlinear systems with unknown inputs. For continuous-time systems, local considerations are used in [19], sliding mode techniques in [20] or LMI-based approaches in [30] taking into account the Lipschitz nature of the nonlinearities. The scarce consideration of nonlinear discrete-time systems with unknown inputs motivates the present work. In this paper we propose a dead-beat delayed functional observer for discrete-time LPV systems with unknown inputs. Let us notice that different terms are

\footnotetext{
GIPSA-lab, Grenoble Campus, 11 rue des Mathématiques, BP 46, 38402 Saint Martin d'Hères Cedex, France. mirko.fiacchini@gipsa-lab.fr

\# Research Center for Automatic Control of Nancy (CRAN), Université de Lorraine, Nancy, France. gilles.millerioux@univ-lorraine.fr
}

used in the literature to define the same concept: dead-beat, finite-time, fast or non-asymptotic.

Closely related works can be cited with their discrepancies. The paper [17] addresses the estimation of a nonlinear function of the state of nonlinear discrete-time systems but the inputs are supposed to be known. Delayed functional observers for discrete-time systems was one of the issues treated in [25] and [29] but the works exclusively deal with linear systems. Finite-time unknown inputs functional observers have been proposed in [15] for singular systems. Finite-time state reconstruction is also closely related to algebraic observability. Concerning this issue, the reader should refer to [13] [2] for continuous nonlinear systems and [16] [11] for linear and polynomial discrete-time systems. However, these works consider the problem of full state reconstruction and partial state reconstruction is not addressed. Algebraic observability was previously addressed in [24] in the context of optimal control as "perfect observability".

The approach proposed in this paper for the design of deadbeat functional observers is based on results of set-theory for control, in particular the concept of invariance. Invariance of a set or a subspace of the state space is related to many classical topics in control, such as stability, Lyapunov theory, constrained control. Invariance and set-theoretic methods in control appeared at the end of the sixties, see the pioneering work [6], and they raised an increasing interest in the last decades, see in particular the recent monograph [7] and references therein for an exhaustive overview. The characterization of invariant subspaces, strongly related also with the properties of controllability and observability, has been treated in [3]-[5]. More recently, invariant subspaces have been used in [1] for similar purposes in the context of LPV systems.

The outline of the paper follows. Section II is devoted to the problem statement. In Section III, a condition based on the notion of inverse system for the dead-beat linear functional observer to exist is given. Two approaches to check the condition in a tractable way are presented in Section IV. The concepts of invariant subspaces and nilpotent semigroups are used. The computational issues are addressed in Section V and an illustrative example is given in Section VI.

Notation: $\mathbb{N}$ is the set of natural numbers. Given $n \in \mathbb{N}$ denote $\mathbb{N}_{n}=\{x \in \mathbb{N}: 1 \leq x \leq n\}$ and $\overline{\mathbb{N}}_{n}=\mathbb{N}_{n} \cup\{0\}$. Denote with $x^{(i)}$ the $i$-th entry of the vector $x$, with $x_{k}$ the realization of the time-dependent vector $x$ at time $k$. Denote with $\mathbf{I}_{m}$ the identity matrix of dimension $m$ and with $\mathbf{0}_{p \times q}$ the matrix in $\mathbb{R}^{p \times q}$ whose entries are zero. Denote with $P_{i}$ the $i$-th element of a set of matrices. We assume the convention that $\prod_{i=0}^{K} P_{i}=$ $P_{K} \ldots P_{0}$. For a matrix $X, X^{\dagger}$ stands for the classical MoorePenrose generalized inverse of $X$. 


\section{Problem StATEMENT}

Consider the discrete-time Linear Parameter Varying (LPV) system obeying

$$
\left\{\begin{array}{l}
x_{k+1}=A\left(\theta_{k}\right) x_{k}+B\left(\theta_{k}\right) u_{k} \\
y_{k}=C\left(\theta_{k}\right) x_{k}+D\left(\theta_{k}\right) u_{k},
\end{array}\right.
$$

where $x_{k} \in \mathbb{R}^{n}$ is the state vector, $u_{k} \in \mathbb{R}^{m}$ is the control input assumed to be unknown, $y_{k} \in \mathbb{R}^{p}$ is the output vector. The matrices $A \in \mathbb{R}^{n \times n}, B \in \mathbb{R}^{n \times m}, C \in \mathbb{R}^{p \times n}$ and $D \in \mathbb{R}^{p \times m}$ depend on the parameter vector $\theta_{k} \in \mathbb{R}^{s}$ belonging to a known set $\Omega_{\theta}$, possibly unbounded.

We are interested in the following issue.

Problem 1: Given $G \in \mathbb{R}^{d \times n}$, provide a condition for the existence of a parameter-dependent function $f_{\theta}$ and $t_{1}, t_{2} \in \mathbb{Z}$ such that

$$
G x_{k}=f_{\theta}\left(y_{k+t_{1}}, \ldots, y_{k+t_{2}}\right),
$$

for every $x_{k} \in \mathbb{R}^{n}$ and every possible realization of the parameter $\theta_{k} \in \Omega_{\theta}$.

As defined in [28], the quantity $G x_{k}$ is called a rank-d linear functional. The function $f_{\theta}$ acts as a dead-beat delayed linear functional observer for reconstructing this functional. Having in mind the different applications mentioned in the introduction, it is noteworthy to point out that this problem is rather general. Indeed, every linear combination of the state can be selected by properly choosing $G$ and then partial and full state reconstruction can be addressed.

The proposed approach to solve Problem 1 rests on the notion of inverse system of (1) which is detailed in the following definition.

Definition 1: An inverse system for (1) is a system for which, when driven by a sequence of output $y_{k}$ of (1), the trajectory of its state vector $\hat{x}_{k}$ coincides with the trajectory of $x_{k}$ (possibly up to a delay $r \geq 0$ referred to as inherent delay, by analogy with the term used in [26] for linear systems) whenever both state vectors share the same initialization.

Define $\mathscr{O}\left(\theta_{k: k+i}\right)=C\left(\theta_{k}\right)$ if $i=0$ and

$$
\mathscr{O}\left(\theta_{k: k+i}\right)=\left[\begin{array}{c}
C\left(\theta_{k}\right) \\
C\left(\theta_{k+1}\right) A\left(\theta_{k}\right) \\
\vdots \\
C\left(\theta_{k+i}\right) \prod_{l=0}^{i-1} A\left(\theta_{k+l}\right)
\end{array}\right], \quad \text { if } i>0,
$$

the vectors

$$
\begin{aligned}
y_{k: k+i} & =\left[y_{k}^{T}, y_{k+1}^{T}, \ldots, y_{k+i}^{T}\right]^{T}, \\
\theta_{k: k+i} & =\left[\theta_{k}^{T}, \theta_{k+1}^{T}, \ldots, \theta_{k+i}^{T}\right]^{T},
\end{aligned}
$$

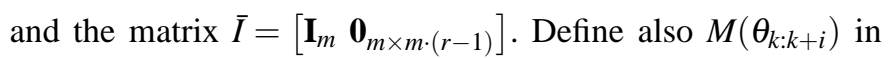
the following recursive way

$$
M\left(\theta_{k: k+i}\right)=\left[\begin{array}{cc}
D\left(\theta_{k}\right) & \mathbf{0}_{p \times m \cdot(i-1)} \\
\mathscr{O}\left(\theta_{k: k+i}\right) B\left(\theta_{k}\right) & M\left(\theta_{k+1: k+i}\right)
\end{array}\right],
$$

with $M\left(\theta_{k: k}\right)=D\left(\theta_{k}\right)$. The following proposition is a straightforward extension of the result given in [27] for switched systems.
Proposition 1: If there exists an integer $r \geq 0$ fulfilling for every possible realization of the parameter $\theta_{k} \in \Omega_{\theta}$

$$
\operatorname{rank} M\left(\theta_{k: k+r}\right)-\operatorname{rank} M\left(\theta_{k+1: k+r}\right)=\operatorname{rank}\left[\begin{array}{c}
B\left(\theta_{k}\right) \\
D\left(\theta_{k}\right)
\end{array}\right],
$$

then the system

$$
\hat{x}_{k+r+1}=P_{\theta}\left(\theta_{k: k+r}\right) \hat{x}_{k+r}+Q_{\theta}\left(\theta_{k: k+r}\right) y_{k: k+r},
$$

with

$$
\begin{aligned}
& P_{\theta}\left(\theta_{k: k+r}\right)=A\left(\theta_{k}\right)-Q\left(\theta_{k: k+r}\right) \mathscr{O}\left(\theta_{k: k+r}\right), \\
& Q_{\theta}\left(\theta_{k: k+r}\right)=B\left(\theta_{k}\right) \bar{I} M^{\dagger}\left(\theta_{k: k+r}\right),
\end{aligned}
$$

is an inverse system for (1).

Proof: Define $e_{k}=x_{k}-\hat{x}_{k+r}$ as the state reconstruction error. Analogously to [27], it can be shown that, from (1) and (3) and provided that (2) is satisfied, the dynamics of the error reads

$$
e_{k+1}=P_{\theta}\left(\theta_{k: k+r}\right) e_{k},
$$

Hence, if $e_{0}=0$, that is $\hat{x}_{r}=x_{0}$, then $e_{k}=0$ and so $\hat{x}_{k+r}=x_{k}$ for every $k>0$, which is in accordance with Definition 1.

\section{DIRECT APPROACH}

The following result yields to characterize a solution to Problem 1.

Proposition 2: If there exists a positive $K \in \mathbb{N}$ and $P_{\theta}\left(\theta_{k+l: k+l+r}\right)$ with $l \in \overline{\mathbb{N}}_{K}$ are such that

$$
G \prod_{l=0}^{K} P_{\theta}\left(\theta_{k+l: k+l+r}\right)=0,
$$

for all $\theta_{k+l: k+l+r} \in \Omega_{\theta}^{r+1}$, for every $l \in \overline{\mathbb{N}}_{K}$, then $G \hat{x}_{k+K+r+1}$ is a function of the outputs $y_{k+j}$ and the parameters $\theta_{k+j}$ with $j \in \overline{\mathbb{N}}_{K+r}$.

Proof: From (3) we have

$\hat{x}_{k+K+r+1}=\prod_{l=0}^{K} P_{\theta}\left(\theta_{k+l: k+l+r}\right) \hat{x}_{k+r}+Q_{\theta}\left(\theta_{k+K: k+K+r}\right) y_{k+K: k+K+r}$ $+\sum_{l=0}^{K-1} \prod_{t=l+1}^{K} P_{\theta}\left(\theta_{k+t: k+t+r}\right) Q_{\theta}\left(\theta_{k+l: k+l+r}\right) y_{k+l: k+l+r}$

and then, whenever (6) holds, $G \hat{x}_{k+K+r+1}$ does not depend on the initial state $\hat{x}_{k+r}$ and reads

$$
\begin{aligned}
& G \hat{x}_{k+K+r+1}=G Q_{\theta}\left(\theta_{k+K: k+K+r}\right) y_{k+K: k+K+r} \\
& +G \sum_{l=0}^{K-1} \prod_{t=l+1}^{K} P_{\theta}\left(\theta_{k+t: k+t+r}\right) Q_{\theta}\left(\theta_{k+l: k+l+r}\right) y_{k+l: k+l+r}
\end{aligned}
$$

From the definition of inverse system, condition (6) implies the following result.

Proposition 3: If $K$ and $P_{\theta}\left(\theta_{k+l: k+l+r}\right)$ with $l \in \overline{\mathbb{N}}_{K}$ are such that (6) holds then $G \hat{x}_{k+K+r+j}=G x_{k+K+j}$ for all $j>0$.

Proof: From (5) and (6), we have that $G e_{k+K+1}=$ $G \prod_{l=0}^{K} P_{\theta}\left(\theta_{k+l: k+l+r}\right) e_{k}=0$ for all $e_{k} \in \mathbb{R}^{n}$, which implies $G \hat{x}_{k+K+r+j}=G x_{k+K+j}$ for all $j>0$.

The following proposition is a direct consequence of Proposition 2, in particular considering the equation (7), and Proposition 3 . 
Proposition 4: If $K$ and $P_{\theta}\left(\theta_{k+l: k+l+r}\right)$ with $l \in \overline{\mathbb{N}}_{K}$ are such that (6) holds then Problem 1 is solved with $f_{\theta}$ given by

$$
\begin{aligned}
& G x_{k}=G \hat{x}_{k+r}=G Q_{\theta}\left(\theta_{k-1: k+r-1}\right) y_{k-1: k+r-1} \\
& \quad+G \sum_{l=0}^{K-1} \prod_{t=l+1}^{K} P_{\theta}\left(\theta_{k+t-K-1: k+t+r-K-1}\right) \\
& \cdot Q_{\theta}\left(\theta_{k+l-K-1: k+l+r-K-1}\right) y_{k+l-K-1: k+l+r-K-1},
\end{aligned}
$$

and the integers fulfill $t_{1}=-K-1$ and $t_{2}=r-1$.

In the remaining part of the paper we provide an approach to check whether the condition (6) is fulfilled and to reformulate Proposition 4 accordingly. First, notice that the parametric dependence of the matrices in (3) and (4) with respect to $\theta_{k: k+r} \in \Omega_{\theta}^{r+1}$ is not linear in general. However, given the set $\Omega_{\theta}$, there always exists a function $p$ such that $P_{\theta}$ depends linearly on the parameter $\rho_{k}$ defined as $\rho_{k}=p\left(\theta_{k: k+r}\right)$. Consider for instance $p$ determined by the functions of $\theta_{k+j}$, with $j \in \overline{\mathbb{N}}_{r}$, appearing in the entries of $P_{\theta}\left(\theta_{k: k+r}\right)$. A set $\Omega_{\rho} \subseteq \mathbb{R}^{q}$ such that $\rho_{k} \in \Omega_{\rho}$ if $\theta_{k: k+r} \in \Omega_{\theta}^{r+1}$ can always be determined as well. To simplify the notation, we define

$$
P\left(\rho_{k}\right)=P_{\theta}\left(\theta_{k: k+r}\right), \quad Q\left(\rho_{k}\right)=Q_{\theta}\left(\theta_{k: k+r}\right), \quad \bar{y}_{k}=y_{k: k+r} .
$$

Following the considerations above, $P\left(\rho_{k}\right)$ can be expressed as

$$
P\left(\rho_{k}\right)=\sum_{i=1}^{q} P_{i} \rho_{k}^{(i)},
$$

for all $\rho_{k} \in \Omega_{\rho}$ and with $\Omega_{\rho}$ subset of $\mathbb{R}^{q}$ and Proposition 4 is reformulated as follows.

Theorem 1: There exists a solution to Problem 1 with $t_{1}=$ $-K-1$ and $t_{2}=r-1$ if there is a positive $K \in \mathbb{N}$ and $P_{i}$, for $i \in \mathbb{N}_{q}$, are such that

$$
G \prod_{k=0}^{K} P_{i_{k}}=0
$$

for all $\mathscr{I}=\left[i_{0}, \ldots, i_{K}\right]^{T} \in \mathbb{N}_{q}^{K+1}$. The function $f_{\theta}$ satisfies

$$
\begin{aligned}
& G x_{k}=G \hat{x}_{k+r}=G Q\left(\rho_{k-1}\right) \bar{y}_{k-1} \\
& +G \sum_{j=0}^{K-1} \sum_{\mathscr{I} \in \mathbb{N}_{q}^{j+1}} \prod_{l=K-j}^{K} \rho_{k+l-K-1}^{\left(i_{l}\right)} P_{i_{l}} Q\left(\rho_{k-j-2}\right) \bar{y}_{k-j-2} .
\end{aligned}
$$

Proof: By definition we have

$$
\begin{aligned}
& G \hat{x}_{k+r+1}=G \sum_{i_{0} \in \mathbb{N}_{q}} \rho_{k}^{\left(i_{0}\right)} P_{i_{0}} \hat{x}_{k+r}+G Q\left(\rho_{k}\right) \bar{y}_{k}, \\
& G \hat{x}_{k+r+2}=G \sum_{\mathscr{I} \in \mathbb{N}_{q}^{2}} \prod_{l=0}^{1} \rho_{k+l}^{\left(i_{l}\right)} P_{i_{l}} \hat{x}_{k+r}+G Q\left(\rho_{k+1}\right) \bar{y}_{k+1} \\
& \quad+G \sum_{\mathscr{I} \in \mathbb{N}_{q}^{1}} \rho_{k}^{\left(i_{0}\right)} P_{i_{0}} Q\left(\rho_{k}\right) \bar{y}_{k}, \\
& \ldots \\
& G \hat{x}_{k+r+K+1}=G \sum_{\mathscr{I} \in \mathbb{N}_{q}^{K+1}} \prod_{l=0}^{K} \rho_{k+l}^{\left(i_{l}\right)} P_{i_{l}} \hat{x}_{k+r}+G Q\left(\rho_{k+K}\right) \bar{y}_{k+K} \\
& \quad+G \sum_{j=0}^{K-1} \sum_{\mathscr{I} \in \mathbb{N}_{q}^{j+1}} \prod_{l=K-j}^{K} \rho_{k+l}^{\left(i_{l}\right)} P_{i_{l}} Q\left(\rho_{k+K-1-j}\right) \bar{y}_{k+K-1-j},
\end{aligned}
$$

where $\mathscr{I}=\left[i_{0}, \ldots, i_{k}\right]^{T} \in \mathbb{N}_{q}^{k+1}$ for all $k \in \overline{\mathbb{N}}_{K}$. If for every $\mathscr{I} \in \mathbb{N}_{q}^{K+1}$ condition (9) holds, then

$$
\begin{aligned}
& G \hat{x}_{k+r+K+1}=G Q\left(\rho_{k+K}\right) \bar{y}_{k+K} \\
& \quad+G \sum_{j=0}^{K-1} \sum_{\mathscr{I} \in \mathbb{N}_{q}^{j+1}} \prod_{l=K-j}^{K} \rho_{k+l}^{\left(i_{l}\right)} P_{i_{l}} Q\left(\rho_{k+K-1-j}\right) \bar{y}_{k+K-1-j} .
\end{aligned}
$$

Finally, based on Propositions 3 and a suitable shift, we get (10) which completes the proof and shows that $f_{\theta}$ involves the output $y_{k+l}$ (and the parameter $\theta_{k+l}$ ) for $-K-1 \leq l \leq r-1$.

The point is that checking condition (9) can be computationally unaffordable since it requires to test the product of many sequences of $P\left(\rho_{k}\right)$, whose number can be exponential in $K$. In the following, two approaches are presented to obtain tractable conditions for (9) to hold.

\section{INVARIANT SET-BASED APPROACH}

The concept of invariant subspaces is recalled here, see the monograph [5] for details.

Definition 2 (Invariant subspace): Given the matrix $A \in$ $\mathbb{R}^{n \times n}$, a subspace $\mathscr{V} \subseteq \mathbb{R}^{n}$ is an invariant if $A \mathscr{V} \subseteq \mathscr{V}$. Given the set of matrices $A(\rho) \subseteq \mathbb{R}^{n \times n}$, defined by $A: \mathbb{R}^{q} \rightarrow \mathbb{R}^{n \times n}$ for $\rho \in \Omega_{\rho}$, a subspace $\mathscr{V} \subseteq \mathbb{R}^{n}$ is a robust invariant if $A(\rho) \mathscr{V} \subseteq \mathscr{V}$, for all $\rho \in \Omega_{\rho}$. Given a subspace $\mathscr{C} \subseteq \mathbb{R}^{n}$, the maximal invariant (resp. robust invariant) subspace contained in $\mathscr{C}$ is given by the sum of all invariant (resp. robust invariant) subspaces contained in $\mathscr{C}$.

In other words, $\mathscr{V}$ is an invariant (resp. robust invariant) subspace if every state trajectory generated by the map $A$ (resp. $A(\rho))$ starting inside it, remains confined within it. The set of invariant (resp. robust invariant) subspaces contained in $\mathscr{C}$ admits a supremum. We recall here a property of the invariant subspaces, see [5].

Property 1 ( [5]): Given a linear transformation $A: \mathbb{R}^{n} \rightarrow$ $\mathbb{R}^{n}$, a subspace $\mathscr{V} \subseteq \mathbb{R}^{n}$ with matrix basis $T \in \mathbb{R}^{n \times g}$ is invariant if and only if there exists a matrix $X \in \mathbb{R}^{g \times g}$ such that

$$
A T=T X .
$$

Clearly the kernel of $A$, denoted $\operatorname{ker}(A)$, is an invariant subspace. Indeed, if $T$ is the basis matrix of $\operatorname{ker}(A)$, it follows that $A T=0$, and then (11) holds merely posing $X=0$. Alternatively seen, if $x \in \operatorname{ker}(A)$ then $A x=0 \in \operatorname{ker}(A)$, and hence $\operatorname{ker}(A)$ is invariant.

Definition 3: Given $P\left(\rho_{k}\right)$ as in (8), define

$$
\mathscr{P}=\bigcap_{i=1}^{q} \operatorname{ker}\left(P_{i}^{T}\right) .
$$

Moreover, denote with $M \in \mathbb{R}^{n \times g}$ a basis matrix of $\mathscr{P}$.

Notice that the definition of $\mathscr{P}$ above involves the transpose of the matrices $P_{i}$ for all $i \in \mathbb{N}_{q}$.

Proposition 5: The set $\mathscr{P}$ as in Definition 3 is an invariant subspace for the LPV system given by $z_{k+1}=P_{T}\left(\rho_{k}\right) z_{k}$ with

$$
P_{T}\left(\rho_{k}\right)=\sum_{i=1}^{q} \rho_{k}^{(i)} P_{i}^{T}=P\left(\rho_{k}\right)^{T} .
$$

Proof: Since $M$ is a basis matrix of $\mathscr{P}$, then $P_{i}^{T} M=0$ for all $i \in \mathbb{N}_{q}$, which implies that $P_{T}\left(\rho_{k}\right) M=0$. From Property $1, \mathscr{P}$ is invariant for the system whose dynamics is given by (12) if and only if there exists $X$ such that $P_{T}\left(\rho_{k}\right) M=M X$, that holds posing $X=0$. 


\section{A. Kernel-based approach}

A sufficient condition for Theorem 1 to hold, then for Problem 1 to admit a solution, follows.

Proposition 6: Consider the system (3), the set $\mathscr{P}$ and the matrix $M$ as in Definition 3. If $G \in \mathbb{R}^{d \times n}$ is such that the columns of $G^{T}$ are in $\mathscr{P}$, i.e. there is $X \in \mathbb{R}^{d \times g}$ such that $G=X M^{T}$, then there exists a solution to Problem 1 with $K=0$.

Proof: From the definition of $\mathscr{P}$, if the rows of $G$ are in $\mathscr{P}$ then $G P_{i}=0$, for all $i \in \mathbb{N}_{q}$. Thus condition (9), sufficient for solving Problem 1, holds with $K=0$.

The result in Proposition 6 is implicitly based on the fact that $\mathscr{P}$ is an invariant for the system (3) as proved in Proposition 5. On the other hand, this condition requires that the state of (3) reaches the invariant subspace in one step, i.e. $G \hat{x}_{k+r+1}=0$. This is obviously restrictive but provides an interesting insight on the problem and a clue on its possible alternative solution.

\section{B. Invariance-based approach}

The definition introduced below is central for what follows.

Definition 4: Given the system (3) with $P\left(\rho_{k}\right)$ as in (8), denote: with $\mathscr{G}$ the maximal robust invariant subspace for $\left\{P_{i}\right\}_{i=1}^{q} \subseteq \mathbb{R}^{n \times n}$ contained in $\operatorname{ker}(G)$; with $g$ the dimension of $\mathscr{G}$; with $\bar{T} \in \mathbb{R}^{n \times g}$ its basis matrix and with $\hat{T} \in \mathbb{R}^{n \times h}$ a matrix such that $T=[\bar{T}, \hat{T}] \in \mathbb{R}^{n \times n}$ is nonsingular.

Based on the results presented in [5], the dynamics on the robust invariant subspace and on its complement can be characterized as follows.

Proposition 7: Given the system (3) with $P\left(\rho_{k}\right)$ as in (8), consider $\mathscr{G}$ and $T$ as in Definition 4 . Then $\mathscr{G}$ is invariant for $P\left(\rho_{k}\right)$ and there exist $\bar{P}\left(\rho_{k}\right) \in \mathbb{R}^{g \times g}, \tilde{P}\left(\rho_{k}\right) \in \mathbb{R}^{g \times h}$ and $\hat{P}\left(\rho_{k}\right) \in \mathbb{R}^{h \times h}$, linear in $\rho_{k}$ and such that:

$$
P\left(\rho_{k}\right)=T\left[\begin{array}{cc}
\bar{P}\left(\rho_{k}\right) & \tilde{P}\left(\rho_{k}\right) \\
\mathbf{0}_{h \times g} & \hat{P}\left(\rho_{k}\right)
\end{array}\right] T^{-1},
$$

for every $\rho_{k} \in \Omega_{\rho}$.

Proof: First notice that, by definition of invariant subspaces, if $\mathscr{G}$ is a robust invariant for $\left\{P_{i}\right\}_{i=1}^{q}$ then it is invariant for every single $P_{i}$. This implies that there exist $\bar{P}_{i} \in \mathbb{R}^{g \times g}, \tilde{P}_{i} \in$ $\mathbb{R}^{g \times h}, \hat{P}_{i} \in \mathbb{R}^{h \times h}$ such that

$$
P_{i}=\left[\begin{array}{ll}
\bar{T} & \hat{T}
\end{array}\right]\left[\begin{array}{cc}
\bar{P}_{i} & \tilde{P}_{i} \\
\mathbf{0}_{h \times g} & \hat{P}_{i}
\end{array}\right]\left[\begin{array}{cc}
\bar{T} & \hat{T}
\end{array}\right]^{-1},
$$

for all $i \in \mathbb{N}_{q}$, being $\bar{T}$ a basis of $\mathscr{G}$. Then (13) holds by posing

$$
\bar{P}\left(\rho_{k}\right)=\sum_{i=1}^{q} \bar{P}_{i} \rho_{k}^{(i)}, \tilde{P}\left(\rho_{k}\right)=\sum_{i=1}^{q} \tilde{P}_{i} \rho_{k}^{(i)}, \hat{P}\left(\rho_{k}\right)=\sum_{i=1}^{q} \hat{P}_{i} \rho_{k}^{(i)},
$$

for all $\rho_{k} \in \Omega_{\rho}$. Thus $\mathscr{G}$ is invariant for $P\left(\rho_{k}\right)$, from (11).

Based on Proposition 7, the following theorem, considered as the central theorem of the paper, can be stated as an alternative to Theorem 1 .

Theorem 2: There exists a solution to Problem 1 with $t_{1}=$ $-K-1$ and $t_{2}=r-1$ if there is a positive $K \in \mathbb{N}$ and $P_{i}$, for $i \in \mathbb{N}_{q}$, are such that

$$
\prod_{k=0}^{K} \hat{P}_{i_{k}}=0,
$$

for all $\mathscr{I}=\left[i_{0}, \ldots, i_{K}\right]^{T} \in \mathbb{N}_{q}^{K+1}$. The function $f_{\theta}$ reads as (10).
Proof: By construction, $\bar{T}$ is a basis of the invariant subspace $\mathscr{G}$ that is contained in $\operatorname{ker}(G)$ then $G \bar{T}=0$. Hence, for all $\mathscr{I}=\left[i_{0}, \ldots, i_{K}\right]^{T} \in \mathbb{N}_{q}^{K+1}$, we have

$$
\begin{aligned}
& G \prod_{k=0}^{K} P_{i_{k}}=G \prod_{k=0}^{K} T\left[\begin{array}{cc}
\bar{P}_{i_{k}} & \tilde{P}_{i_{k}} \\
\mathbf{0}_{h \times g} & \hat{P}_{i_{k}}
\end{array}\right] T^{-1} \\
& =\prod_{k=0}^{K} G[\bar{T}, \hat{T}]\left[\begin{array}{cc}
\bar{P}_{i_{k}} & \tilde{P}_{i_{k}} \\
\mathbf{0}_{h \times g} & \hat{P}_{i_{k}}
\end{array}\right] T^{-1}=\left[\mathbf{0}_{d \times g}, G \hat{T} \prod_{k=0}^{K} \hat{P}_{i_{k}}\right] T^{-1}=0 .
\end{aligned}
$$

Thus condition (9) is satisfied for all $\mathscr{I}=\left[i_{0}, \ldots, i_{K}\right]^{T} \in \mathbb{N}_{q}^{K+1}$, which is sufficient for the existence of a solution to Problem 1. Finally, the expression of $f_{\theta}$ is obtained by following along the same lines as in the proof of Theorem 1.

The function $f_{\theta}$ stands for the expected dead-beat delayed linear functional observer written in terms of $P\left(\rho_{k}\right)$ and $Q\left(\rho_{k}\right)$. Finally, we are in position for giving a tractable approach for checking (14). To this end, let us recall the concept of nilpotent semigroups.

Definition 5 (Semigroup): A semigroup $\mathscr{S}$ is a set together with an associative internal law.

It is said to be finite if it has a finite number of elements. If $\mathscr{S}$ is a set of matrices, the associative internal law is the matrix multiplication and there exists an absorbing element which is the null matrix. In such a case, we can define a nilpotent semigroup of matrices.

Definition 6 (Nilpotent semigroup): A semigroup $\mathscr{S}$ of matrices with the multiplication law is said to be nilpotent if any product involving a finite number $t \in \mathbb{N}$ of its elements (possibly the same element) is always equal to the null matrix. The smallest integer $t$ is called the class of nilpotency of $\mathscr{S}$.

Then, the relation (14) in Theorem 2 means that $\hat{P}_{i}$ with $i \in \mathbb{N}_{q}$ generate a nilpotent semigroup of class $t=K+1$. As a direct consequence, the following computation-oriented result follows.

Corollary 1: If $\hat{P}_{i}$ for all $i \in \mathbb{N}_{q}$, as defined in Proposition 7, generate a nilpotent semigroup of class $t$ then Problem 1 is solved with $K=t-1$. The explicit function $f_{\theta}$ is given by (10) with $t_{1}=-t$ and $t_{2}=r-1$.

Remark 1: The class of nilpotency $t$ is smaller than or equal to $h$, that is the dimension of the subspace complementary to $\mathscr{G}$ in $\mathbb{R}^{n}$. Then, the bigger the dimension of $\mathscr{G}$, the smaller such a bound, in general. Moreover, notice that for lower values of $d$, number of rows of $G$, the dimension of $\mathscr{G}$ can be higher and then the dimension of matrices $\hat{P}_{i}$ is smaller. This is reasonable, as the smaller is the partial state to be estimated through the inverse system, the smaller is the invariant subspace, on which the state projection must converge to 0 in finite time.

\section{Computational issues}

In this section, we first give some insights on the computational procedures for obtaining the maximal robust invariant subspace and the matrices $\hat{P}_{i}$ with $i \in \mathbb{N}_{q}$ of Proposition 7 involved in Theorem 2 . Then some considerations on the condition for the matrices $\hat{P}_{i}$ with $i \in \mathbb{N}_{q}$ to generate a nilpotent semigroup, as stated in Corollary 1 , are provided.

According to Definition 2, a subspace $\mathscr{V}$ is invariant for a linear system if its image through the linear transformation is contained in $\mathscr{V}$. Well established results on how to generate 
invariant subspaces have been presented in literature, see in particular [5]. Interestingly for our purpose, the algorithm for computing invariant subspaces for LPV systems are directly obtained from those concerning linear systems, see [1], [5]. Every robust invariant subspace (then also the maximal one) contained in $\mathscr{V}$ for $\left\{A_{i}\right\}_{i=1}^{q}$ is a robust invariant subspace also for $A\left(\Omega_{\rho}\right)=\left\{A(\rho)=\sum_{i=1}^{q} A_{i} \rho^{(i)}: \rho \in \Omega_{\rho}\right\}$ for all $\Omega_{\rho} \subseteq \mathbb{R}^{q}$, as proved in Proposition 7. Given $\mathscr{A} \subseteq \mathbb{R}^{n \times n}$, every robust invariant for any $A\left(\Omega_{\rho}\right)$ such that $\mathscr{A} \subseteq A\left(\Omega_{\rho}\right)$ is invariant also for $\mathscr{A}$. Clearly, the smaller is the set $A\left(\Omega_{\rho}\right)$ containing $\mathscr{A}$, the closer are the sets of invariant subspaces for $A\left(\Omega_{\rho}\right)$ and for $\mathscr{A}$. The following algorithm provides a robust invariant subspace contained in $\mathscr{V}$ for every $\mathscr{A} \subseteq \mathbb{R}^{n \times n}$ whose elements are linear combinations of the matrices $A_{i}$ with $i \in \mathbb{N}_{q}$.

Algorithm 1: Given the subspace $\mathscr{V} \subseteq \mathbb{R}^{n}$ and the set of matrices $\left\{A_{i}\right\}_{i=1}^{q} \subseteq \mathbb{R}^{n \times n}$, the maximal robust invariant subspace for $\left\{A_{i}\right\}_{i=1}^{q}$ contained in $\mathscr{V}$ is $\mathscr{J}_{n}$ provided by:

$$
\begin{aligned}
& \mathscr{J}_{0}=\mathscr{V}, \\
& \mathscr{J}_{k+1}=\mathscr{J}_{0} \cap \bigcap_{i \in \mathbb{N}_{q}} A_{i}^{-1} \mathscr{J}_{k}, \quad k=0, \ldots, n-1 .
\end{aligned}
$$

Remark 2: It can be proved that the same sequence of subspaces $\mathscr{J}_{k}$ with $k=0, \ldots, n$ is generated by the following iteration

$$
\mathscr{J}_{k+1}=\mathscr{J}_{k} \cap \bigcap_{i \in \mathbb{N}_{q}} A_{i}^{-1} \mathscr{J}_{k}, \quad k=0, \ldots, n-1 .
$$

Remark 3: Notice that, with a slight abuse of notation, the meaning of the symbol $A_{i}^{-1}$ does not denote the inverse of matrix $A_{i}$, but it is the operator that associates to a subspace its inverse image. That is, given the subspace $\mathscr{J}_{k} \subseteq \mathbb{R}^{n}$ one have $A_{i}^{-1} \mathscr{J}_{k}=\left\{x \in \mathbb{R}^{n}: A_{i} x \in \mathscr{J}_{k}\right\}$, which is defined also for singular and nilpotent matrices.

Remark 4: Algorithm 1, either implementing the iteration (15) or (16), is based, substantially, on the computation of the intersection between subspaces and of the inverse image of a subspace. We provide hereafter a sketch of the procedure. Consider two subspaces $\mathscr{V} \subseteq \mathbb{R}^{n}$ and $\mathscr{U} \subseteq \mathbb{R}^{n}$, whose basis matrices are $V \in \mathbb{R}^{n \times v}$ and $U \in \mathbb{R}^{n \times u}$. The subspace $\mathscr{V} \cap A^{-1} \mathscr{U}$ is given by the vectors $x \in \mathbb{R}^{n}$ that can be expressed as a linear combination of the columns of $V$ and their image through $A$ as a linear combination of those of $U$. Then, in practice, $x \in$ $\mathscr{V} \cap A^{-1} \mathscr{U}$ if there exist $y \in \mathbb{R}^{v}$ and $z \in \mathbb{R}^{u}$ such that $x=V y$ and $A x=U z$, that means such that $A V y=U z$. Hence, $\mathscr{V} \cap A^{-1} \mathscr{U}$ can be obtained by computing the kernel of $[A V, U]$.

As for the computational aspects regarding the nilpotency property, involved in Corollary 1, we must recall the Levitsky's theorem (Theorem 2.1.7 stated in [23]).

Theorem 3 (Levitsky's theorem): Any semigroup of nilpotent matrices can be triangularized.

Thus, all the matrices of a same nilpotent semigroup can be triangularized by means of a common change of basis. The triangularization can be performed for example with the algorithm given in [14] and it leads to a substantial reduction of the computational complexity, see [21] or a detailed investigation in [22].

\section{Illustrative EXAMPLE}

The example is devoted to the problem of rewriting a nonlinear discrete-time systems as an LPV system with ac- cessible time-varying parameter $\theta_{k}$. Such a problem has been investigated in [9] and called "exact LPV description". It is noteworthy to stress that LPV systems permit to model a wide class of nonlinear systems. Indeed, it is sufficient, for instance, that the nonlinear dynamical and output functions, respectively denoted $f$ and $h$, are differentiable and such that $f(0,0)=0$ and $h(0,0)=0$ for having equivalence between the two models, see [8]. In case that the parameter is not accessible, the LPV model can be considered as a Linear Difference Inclusion (LDI) system. When the input is assumed to be unknown as it holds here, the problem is the search for a suitable function $\theta_{k}$ which can be expressed in terms of the output over a finite number of time-steps. In general, several such functions may exist and a possible approach is to resort to a functional observer, as illustrated in the following example.

Consider the controlled nonlinear system given by

$$
\left\{\begin{array}{l}
x_{k+1}^{(1)}=-4 x_{k}^{(1)}+x_{k}^{(2)}, \\
x_{k+1}^{(2)}=3\left(x_{k}^{(1)}\right)^{2}+2 x_{k}^{(2)} x_{k}^{(1)}+x_{k}^{(3)}+u_{k}, \\
x_{k+1}^{(3)}=-2 x_{k}^{(1)}+u_{k}, \\
y_{k}=x_{k}^{(1)}
\end{array}\right.
$$

where $u_{k} \in \mathbb{R}$ is the control supposed to be unknown. First, we choose $\theta_{k}=x_{k}^{(1)}$. The system can be rewritten as (1) with $A\left(\theta_{k}\right)=\left[\begin{array}{ccc}-4 & 1 & 0 \\ 3 \theta_{k} & 2 \theta_{k} & 1 \\ -2 & 0 & 0\end{array}\right], B\left(\theta_{k}\right)=\left[\begin{array}{l}0 \\ 1 \\ 1\end{array}\right], C\left(\theta_{k}\right)=\left[\begin{array}{lll}1 & 0 & 0\end{array}\right], D\left(\theta_{k}\right)=0$.

Clearly, the problem of exact LPV description of (17) is trivially solved by such a function $\theta_{k}$ since $\theta_{k}=x_{k}^{(1)}=y_{k}$ and so is accessible from the output.

Alternatively, choosing $\theta_{k}=3 x_{k}^{(1)}+2 x_{k}^{(2)}$, the system (17) results as in (1) with

$$
A\left(\theta_{k}\right)=\left[\begin{array}{ccc}
-4 & 1 & 0 \\
\theta_{k} & 0 & 1 \\
-2 & 0 & 0
\end{array}\right], B\left(\theta_{k}\right)=\left[\begin{array}{l}
0 \\
1 \\
1
\end{array}\right], C\left(\theta_{k}\right)=\left[\begin{array}{lll}
1 & 0 & 0
\end{array}\right], D\left(\theta_{k}\right)=0 .
$$

Now, we must check whether $\theta_{k}$ is accessible from the output. To this end, we search for a dead-beat linear functional observer to estimate $\theta_{k}$, if it exists. The quantity $G x_{k}$ with $G=[3,2,0]$ is the rank-1 linear functional to be estimated. It turns out that (2) is fulfilled with $r=2$. Hence, the related inverse system (3) is characterized (see (4)) by the parameterdependent matrix

$$
P_{\theta}\left(\theta_{k: k+2}\right)=\left[\begin{array}{ccc}
-4 & 1 & 0 \\
-16 & 4 & 0 \\
-14-\theta_{k} & 4 & -1
\end{array}\right]
$$

that is affine in $\theta_{k} \in \mathbb{R}$. Here, the function $p$ such that the matrix $P_{\theta}\left(\theta_{k: k+2}\right)$ depends linearly on a parameter $\rho_{k}$ is merely $\rho_{k}=p\left(\theta_{k: k+2}\right)=\left[1, \theta_{k}\right]^{T}$. The matrices $P_{i}$, as in (8), are

$$
P_{1}=\left[\begin{array}{ccc}
-4 & 1 & 0 \\
-16 & 4 & 0 \\
-14 & 4 & -1
\end{array}\right], \quad P_{2}=\left[\begin{array}{ccc}
0 & 0 & 0 \\
0 & 0 & 0 \\
-1 & 0 & 0
\end{array}\right]
$$

Consider Problem 1 with $G=[3,2,0]$, whose kernel basis is given by the vectors $[-2 / 3,1,0]^{T}$ and $[0,0,1]^{T}$. To check whether Theorem 2 is fulfilled, we compute the matrices $\hat{P}_{i}$, 
as explained in Section V. Algorithm 1 generates a sequence of subspaces $\mathscr{J}_{i}$ and provides the maximal robust invariant subspace $\mathscr{J}_{n}$ in $\operatorname{ker}(G)$, whose basis is $\bar{T}=[0,0,1]^{T}$. Considering the canonical basis matrix $T$, we obtain the matrices $\hat{P}_{i}$ as defined in Proposition 7

$$
\hat{P}_{1}=\left[\begin{array}{cc}
-4 & 1 \\
-16 & 4
\end{array}\right], \quad \hat{P}_{2}=\left[\begin{array}{ll}
0 & 0 \\
0 & 0
\end{array}\right]
$$

Corollary 1 is fulfilled since $\hat{P}_{1}$ and $\hat{P}_{2}$ are both nilpotent (necessary condition) and generate a nilpotent semigroup with class of nilpotency $t=2$. Hence, Problem 1 is solved with $K=1$ and the explicit solution reads

$$
G x_{k}=G \hat{x}_{k+2}=11 y_{k}+2 y_{k+1} \text {. }
$$

As the expression (19) does not depend on $\theta_{k}$, it is a dead-beat delayed linear functional observer suitable to provide an exact LPV description of (17), replacing $\theta_{k}$ by $11 y_{k}+2 y_{k+1}$ in (18).

\section{CONCLUSIONS}

An approach for designing a dead-beat functional observer with unknown inputs for discrete-time LPV systems has been presented. Existence conditions have been derived. They are based on the notion of inverse system, invariant subspaces and nilpotent semigroups. The proof is constructive and an explicit formulation of the observer is provided. The results concern a large class of problems in control, including set-membership analysis or worst-case robust design.

\section{REFERENCES}

[1] G. Balas, J. Bokor, and Z. Szabó. Invariant subspaces for LPV systems and their applications. IEEE Transaction on Automatic Control, 48:2065-2069, 2003.

[2] J-P. Barbot, M. Fliess, and T. Floquet. An algebraic framework for the design of nonlinear observers with unknown inputs. In Proc. of the 46th IEEE Conference on Decision and Control (CDC'07), pages 384-389, December 2007.

[3] G. Basile and G. Marro. Controlled and conditioned invariant subspaces in linear system theory. Journal of Optimization Theory and Applications, 3:306-315, 1969.

[4] G. Basile and G. Marro. On the robust controlled invariant. Systems and Control Letters, 9:191-195, 1987.

[5] G. Basile and G. Marro. Controlled and Conditioned Invariants in Linear System Theory. Prentice Hall, 1992.

[6] D. P. Bertsekas. Infinite-time reachability of state-space regions by using feedback control. IEEE Transactions on Automatic Control, 17:604-613, 1972.

[7] F. Blanchini and S. Miani. Set-Theoretic Methods in Control. Birkhäuser, 2008.

[8] S. Boyd, L. El Ghaoui, E. Feron, and V. Balakrishnan. Linear Matrix Inequalities in system and control theory. SIAM, 1994.

[9] F. Bruzelius. Linear Parameter-Varying Systems: an approach to gain scheduling. $\mathrm{PhD}$ thesis, Department of Signals and Systems, Chalmers University of Technology, Goteborg, Sweden, 2004.

[10] C. T. Chen. Linear System Theory and Design. Oxford Series in Electrical and Computer Engineering, 1984.

[11] J. Daafouz, M. Fliess, and G. Millérioux. Une approche intrinsèque des observateurs linéaires à entrées inconnues. In Proc. of the Conférence Internationale Francophone d'Automatique, Bordeaux, May 2006.

[12] M. Darouach. Existence and design of functional observers for linear systems. IEEE Transactions on Automatic Control, 45(5):940-943, 2000.

[13] S. Diop and M. Fliess. Nonlinear observability, identifiability and persistent trajectories. In Proc. of the 30th IEEE Conference on Decision and Control (CDC'91), Brigthon, England, December 1991.

[14] C. Dubi. An algorithmic approach to simultaneous triangularization. Linear Algebra and its Applications, 430(11-12):2975 - 2981, 2009.
[15] J.-E. Feng. Finite time functional observers for discrete-time singular systems with unknown inputs. In Proc. of the 29th Chinese Control Conference (CCC 2010), Beijing, China, 2010.

[16] R. Germundsson and K. Forsman. A constructive approach to algebraic observability. In Proc. of the 30th IEEE Conference on Decision and Control (CDC'91), Brighton, UK, December 1991.

[17] I. Karafyllis and C. Kravaris. On the observer problem for discrete-time control systems. IEEE Transactions on Automatic Control, 52(1), 2007.

[18] D. G. Luenberger. Observers for multivariable systems. IEEE Transactions on Automatic Control, 11(2):190-197, 1966.

[19] R. Ben Messaoud, N. Zanzouri, and M. Ksouri. Local feedback unknown input observer for nonlinear systems. International Journal of Innovative Computing, Information and Control, 8(2):1145-1154, 2010.

[20] N. Nikraz. Sliding mode functional observers for classes of linear and non-linear systems. $\mathrm{PhD}$ thesis, School of Electrical, Electronic and Computer Engineering, University of Western Australia, 2010.

[21] J. Parriaux and G. Millérioux. Nilpotent semigroups for the characterization of flat outputs of discrete-time switched linear and LPV systems. In Proc. of the 51th IEEE Conference on Decision and Control, pages 4505-4510, Maui, Hawaii, USA, December 2012.

[22] J. Parriaux and G. Millérioux. Nilpotent semigroups for the characterization of flat outputs of switched linear and LPV discrete-time systems. Systems and Control Letters, To be published.

[23] H. Radjavi and P. Rosenthal. Simultaneous Triangularization. Springer, 2000.

[24] D. Rappaport and L. M. Silverman. Structure and stability of discretetime optimal systems. IEEE Transactions on Automatic Control, 16(3):227-233, 1971.

[25] A. Saberi, A. Stoorvogel, and P. Sannuti. Exact, almost and optimal input decoupled (delayed) observers. International Journal of Control, 73(7):552-581, 2000.

[26] M. K. Sain and J. L. Massey. Invertibility of linear time-invariant dynamical systems. IEEE Transactions on Automatic Control, 14:141$149,1969$.

[27] S. Sundaram and C. Hadjicostis. Designing stable inverters and state observers for switched linear systems with unknown inputs. In Proc. of the 45th IEEE Conference on Decision and Control (CDC'06), San Diego, CA, USA, December 2006.

[28] S. Sundaram and C. Hadjicostis. Delayed observers for linear systems with unknown inputs. IEEE Transactions on Automatic Control, 52(2):334 - 339, 2007.

[29] S. Sundaram and C. Hadjicostis. Partial state observers for linear systems with unknown inputs. Automatica, 44(12):3126-3132, 2008.

[30] H. Trinh and T. Fernando. Functional Observers for Dynamical Systems, volume 420 of Lecture Notes in Control and Information Sciences. Springer, 2012. 